\title{
Joint Spectrum Partition and User Association in Multi-Tier Heterogeneous Networks
}

\author{
Yicheng Lin and Wei Yu \\ Department of Electrical and Computer Engineering \\ University of Toronto, Toronto, Ontario, Canada \\ Email: \{ylin,weiyu\}@ comm.utoronto.ca
}

\begin{abstract}
The joint spectrum partition and user association problem for multi-tier heterogeneous networks is studied in this paper, where disjoint spectrums are allocated among tiers and users are associated with each tier with a biased received power. The random placement of base-stations (BSs) of different tiers are modeled using stochastic geometry, which accounts for their practical deployment and also makes analysis tractable. We derive an upper bound of the average user proportional fair utility based on the user coverage rate, from which we formulate a network utility maximization problem. The optimization of the proposed utility bound shows that the optimal spectrum allocation for each BS tier matches the average proportion of users associated with that tier. The solution to the optimization problem also provides closed-form expressions for the optimal user associated bias factors. Compared to system-level optimization solutions based on specific network topology and channel realization, our offline analytical approach offers deployment insights. Simulation results demonstrates the effectiveness of the proposed approach.
\end{abstract}

\section{INTRODUCTION}

Future wireless networks are expected to have exploding mobile date transmission demands, which could be difficult to satisfy by traditional single-tier macro cellular networks. Heterogeneous architecture is proposed to be one solution to this problem, where various types of low-power access nodes such as micro, pico, femto base-stations (BSs) are deployed in addition to the macro BSs to form a multitier topology with a large quantity of small cells. While the macro cells offer basic long-range coverage, the small cells provide short-range but high-quality communication to nearby users. The system parameters such as transmission powers and deployment densities are distinct across BS tiers.

This paper addresses the joint load balancing and interference mitigation problem in a multi-tier network. Our goal is to maximize a utility function that is averaged over network topologies and channel realizations. Such a utility is determined by the user-BS association and the specrum allocation among tiers. In the following, we discuss the system modeling assumptions of this paper, along with a literature review.

Biased user association. Associating users to their nearby BSs with the maximum received power is not necessarily optimal in multi-tier networks, since in this case most users tend to connect to high-power macro BSs. Despite many dynamic and complex approaches to user association available in the literature, this paper adopts the simple but efficient cell range expansion scheme, also known as the biased user

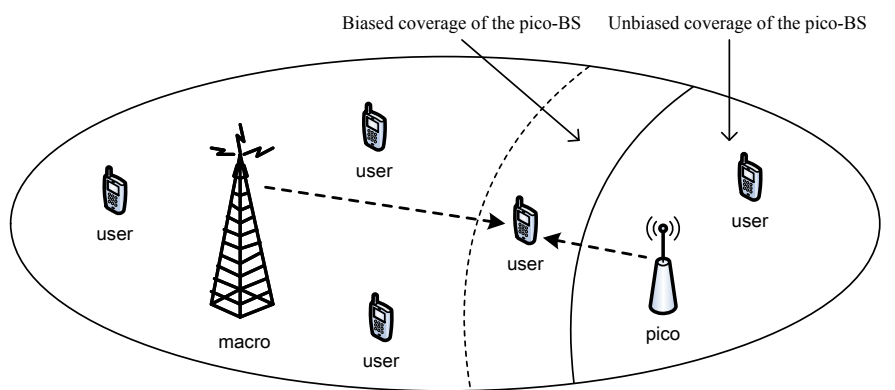

Fig. 1. An example of 2-tier heterogeneous network with cell range extension.

association [1], where each BS is assigned a bias factor, and each user is associated with the BS with the maximum received power weighted by its bias. In this way, traffic can be effectively off-loaded to lower-power nodes by setting a (larger) power bias term towards them. See Fig. 1 as an illustration of 2-tier network with biased association. Note that the bias factors are assumed to be different across tiers but the same within a tier for simplicity, as BSs within a tier are assumed to have approximately the same load. The bias should be properly designed such that all the users receive adequate quality of service, i.e., it should achieve a tradeoff between signal quality from the users' perspective and load balancing from the BSs' perspective. In contrast to most previous works where bias factors are empirically determined, the optimal bias in terms of SIR and rate coverage is determined in [2], [3].

Disjoint spectrum partition. The interference problem is exacerbated when cell range expansion is considered, as users that are offloaded to small cells experience larger-than-usual interferences from macro cells. By partitioning the available spectrum into disjoint portion for each BS tier as proposed in [4], [5], cross-tier interference can be avoided. This greatly reduces the complexity of interference management especially in an irregular topology. However, how to properly divide the spectrum still remains an open issue.

The joint spectrum partition and user association problem is studied in [3], [6]. The coverage probability and user rate are analyzed in [3], but [3] is limited to a two-tier topology and it does not perform optimization over the network parameters. In [6], the spectrum partition and user association are optimized for a general $K$-tier network in terms of user rate, with a constraint on the fraction of spectrum that each tier can get. 
A similar problem is studied in [7], where stochastic frequency reuse instead of disjoint spectrum is assumed among BS tiers.

This paper intends to numerically compute the optimal fraction of spectrum allocated to, and the optimal bias factor of each BS tier in a $K$-tier network. For tractability, we use a random point process to account for the irregular deployment of the heterogeneous small BSs. We adopt a definition of user coverage rate, based on which a closed-form upper bound of the average user proportional fair utility is derived using tools from stochastic geometry. By optimizing the proposed utility function, we show that the optimal proportion of spectrum allocated to each BS tier should match the optimal proportion of users associated with that tier on average. Further, this proportion can be computed in closed form. The optimal user association bias can then be determined subsequently. Simulation results are provided to validate our analysis.

\section{SySTEM MODEL}

\section{A. Multi-Tier Network Topology and Propagation Model}

We consider a heterogeneous network with $K$ tiers, where BSs in the $k$-th tier is modeled as a two dimensional independent homogeneous Poisson point process (PPP) $\Phi_{k}$ with intensity $\lambda_{k}$. Without loss of generality, we assume $\lambda_{1}<\lambda_{2}<$ $\ldots<\lambda_{K}$. Users are assumed to form an independent PPP $\Phi_{u}$ with intensity $\lambda_{u}$. The transmit power of the $k$-th tier BSs is $P_{k}$, and the pathloss exponent is $\alpha$ for all tiers $(\alpha>2$ is assumed to ensure that the expected total noise is finite [8]).

\section{B. Biased User Association}

A user is associated with the $k$-th BS tier with the largest biased received power

$$
P_{k}\left(\min _{\mathcal{L}_{i} \in \Phi_{k}} \ell_{k, i}\right)^{-\alpha} B_{k} \geq P_{j}\left(\min _{\mathcal{L}_{i} \in \Phi_{j}} \ell_{j, i}\right)^{-\alpha} B_{j}, \forall j,
$$

where $\ell_{k, i}$ is the distance from the $i$-th BS in the $k$-th tier to the user, $\mathcal{L}_{i}$ is the BS location and $B_{k}$ is the bias factor of the $k$-th tier.

Denote $A_{k}$ as the probability of a user being associated with BSs in the $k$-th tier. As in [9], we have

$$
A_{k}=\frac{\lambda_{k}\left(P_{k} B_{k}\right)^{2 / \alpha}}{\sum_{j=1}^{K} \lambda_{j}\left(P_{j} B_{j}\right)^{2 / \alpha}} .
$$

We can also recover $\left\{B_{k}\right\}_{\forall k}$ from $\left\{A_{k}\right\}_{\forall k}$. Note that the values of $\left\{B_{k}\right\}_{\forall k}$ are not unique as the scaled bias factors do not affect the user association results. We can choose to normalize the bias factor of the $K$-th tier (the tier with the largest intensity) to unit, i.e, setting $B_{K}=1$, and after some manipulations we have

$$
B_{k}=\left(\frac{\lambda_{K} A_{k}}{\lambda_{k} A_{K}}\right)^{\alpha / 2} \frac{P_{K}}{P_{k}} .
$$

\section{Orthogonal Partition of Spectrum Among Tiers}

We assume that the total system spectrum $W$ is orthogonally partitioned for each tier, in order to avoid inter-tier interference. The fraction of spectrum allocated for the $k$-th tier is denoted as $\eta_{k}$, which satisfies the relation $\sum_{k=1}^{K} \eta_{k}=1$.

\section{Coverage Probability and User Coverage Rate}

The coverage probability of a user associated with the $k$-th tier is defined by the signal-to-interference ratio (SIR) of that tier, i.e.

$$
C_{k}=\mathbb{P}\left(\operatorname{SIR}_{k}>\tau_{k}\right),
$$

where $\tau_{k}$ is the given target SIR of the $k$-th tier, which is determined by the physical-layer requirement such as the target bit error rate (BER). We assume that the noise is negligible compared with the interference, so the SIR of the $k$-th tier can be expressed as

$$
\operatorname{SIR}_{k}=\frac{P_{k} \ell_{k, 0}^{-\alpha} g_{k, 0}}{I_{\Phi_{k}}}
$$

and interferences coming from other BSs of the $k$-th tier are expressed as

$$
I_{\Phi_{k}}=\sum_{\mathcal{L}_{i} \in \Phi_{k} \backslash \mathrm{BS}_{k, 0}} P_{k} \ell_{k, i}^{-\alpha} g_{k, i}
$$

where $g_{k, i}$ is the exponentially distributed channel power gain from the $i$-th $\mathrm{BS}$ in the $k$-th tier. Let the index of the serving BS be 0 , and denote $\mathrm{BS}_{k, 0}$ as the serving BS in the $k$-th tier.

For simplicity of analysis, only one target SIR is assumed for each tier in this paper, which corresponds to the case that only one modulation and coding scheme is available for use. In this case, the spectrum efficiency of the user associated with the $k$-th $\mathrm{BS}$ tier has a binary form (in nats $/ \mathrm{s} / \mathrm{Hz}$ )

$$
r_{k}=\log \left(1+\tau_{k}\right) \mathbf{1}\left(\operatorname{SIR}_{k}>\tau_{k}\right),
$$

where $1(\cdot)$ is the indicator function. By summing across the spectrum $\beta_{k}$ that is allocated to this user, the user coverage rate is proportional to the fraction of frequency spectrum that yields non-zero rate. The average of this rate is

$$
R_{k}=\beta_{k} \mathbb{E}\left(r_{k}\right)=\beta_{k} C_{k} \log \left(1+\tau_{k}\right) .
$$

\section{UTILITY-ORIENTED JOINT OPTIMIZATION OF Spectrum Partition and User Association}

In this section, we first derive the average utility of a typical (randomly chosen) user, based on which we formulate and solve the joint optimization problem with regard to the spectrum partition and user association bias.

\section{A. Average User Utility}

The average utility of the typical user is

$$
U=\sum_{k=1}^{K} A_{k} U_{k},
$$

where $U_{k}$ is the average user utility given that the typical user is associated with the $k$-th tier BS. We adopt the commonly used proportional fair metric for the utility as

$$
\begin{aligned}
U_{k} & =\mathbb{E}\left[\log \left(R_{k}\right)\right] \\
& =\log \left[\log \left(1+\tau_{k}\right)\right]+\mathbb{E}\left[\log \left(\beta_{k}\right)\right]+\mathbb{E}\left[\log \left(C_{k}\right)\right] .
\end{aligned}
$$

The use of the logarithm utility separates the computation of the expected $\beta_{k}$ and $C_{k}$, although they are not independent. 
The average logarithm of per-user spectrum is difficult to capture, we resort to its upper bound based on the concavity of the logarithm function

$$
\begin{gathered}
\mathbb{E}\left[\log \left(\beta_{k}\right)\right]<\log \left[\mathbb{E}\left(\beta_{k}\right)\right]=\log \left[\mathbb{E}\left(\frac{W \eta_{k}}{n_{k}+1}\right)\right] \\
\stackrel{(a)}{=} \log \left(\frac{W \eta_{k} \lambda_{k} q_{k}}{A_{k} \lambda_{u}}\right), \text { where } q_{k}=1-\left(1+\frac{A_{k} \lambda_{u}}{3.5 \lambda_{k}}\right)^{-3.5},
\end{gathered}
$$

where $n_{k}$ is the number of users associated with the serving BS in the $k$-th tier other than the typical user. Using Proposition 2 in [10] for single-tier networks, and replacing the user intensity by $A_{k} \lambda_{u}$ for tier- $k$, (a) is derived since

$$
\mathbb{E}\left(\frac{1}{n_{k}+1}\right)=\frac{\lambda_{k}}{A_{k} \lambda_{u}}\left[1-\left(1+\frac{A_{k} \lambda_{u}}{3.5 \lambda_{k}}\right)^{-3.5}\right] \text {. }
$$

The expected logarithm of the coverage probability is averaged over the user location, BS locations, as well as the interference channel

$$
\begin{aligned}
& \mathbb{E}_{\Phi_{u}, \Phi_{k}, \mathbf{g}}\left[\log \left(C_{k}\right)\right] \\
& =\int_{0}^{\infty} \mathbb{E}_{\Phi_{k}, \mathbf{g}}\left[\log \left(C_{k}\right) \mid \ell_{k, 0}=x\right] f_{\ell_{k}, 0}(x) \mathrm{d} x
\end{aligned}
$$

Under the biased user association, the probability density function (PDF) of the distance between the typical user and its serving $\mathrm{BS}$ in the $k$-th tier is given as in [9]

$$
\begin{aligned}
f_{\ell_{k}, 0}(x) & =\frac{2 \pi \lambda_{k}}{A_{k}} x \exp \left[-\pi x^{2} \sum_{j=1}^{K} \lambda_{j}\left(\frac{P_{j} B_{j}}{P_{k} B_{k}}\right)^{2 / \alpha}\right] \\
& \stackrel{(b)}{=} \frac{2 \pi \lambda_{k}}{A_{k}} x \exp \left(-\pi x^{2} \frac{\lambda_{k}}{A_{k}}\right),
\end{aligned}
$$

where (b) is obtained by plugging (2).

Conditioned on the distance $\ell_{k, 0}=x$ between the typical user and its associated $\mathrm{BS}$ in the $k$-th tier

$$
\begin{aligned}
& \mathbb{E}_{\Phi_{k}, \mathbf{g}}\left[\log \left(C_{k}\right) \mid \ell_{k, 0}=x\right] \\
= & \mathbb{E}_{\Phi_{k}, \mathbf{g}}\left\{\log \left[\mathbb{P}\left(\operatorname{SIR}_{k}>\tau_{k}\right)\right] \mid \ell_{k, 0}=x\right\} \\
= & \mathbb{E}_{\Phi_{k}, \mathbf{g}}\left\{\log \left[\mathbb{P}\left(g_{k, 0}>\tau_{k} P_{k}^{-1} x^{\alpha} I_{\Phi_{k}}\right)\right] \mid \ell_{k, 0}=x\right\} \\
\stackrel{(c)}{=} & \mathbb{E}_{\Phi_{k}, \mathbf{g}}\left\{\log \left[\exp \left(-\tau_{k} P_{k}^{-1} x^{\alpha} I_{\Phi_{k}}\right)\right] \mid \ell_{k, 0}=x\right\} \\
= & -\tau_{k} P_{k}^{-1} x^{\alpha} \mathbb{E}_{\Phi_{k}, \mathbf{g}}\left(I_{\Phi_{k}} \mid \ell_{k, 0}=x\right),
\end{aligned}
$$

where in (c) we assume $g_{k, 0} \sim \exp (1)$ is Rayleigh distributed with unit variance. The downlink mean interference is

$$
\begin{aligned}
\mathbb{E}_{\Phi_{k}, \mathbf{g}}\left(I_{\Phi_{k}} \mid \ell_{k, 0}=x\right) & =P_{k} \mathbb{E}_{\Phi_{k}}\left(\sum_{\mathcal{L}_{i} \in \Phi_{k} \backslash \mathrm{BS}_{k, 0}} \ell_{k, i}^{-\alpha} \mid \ell_{k, 0}=x\right) \\
& \stackrel{(d)}{=} 2 \pi \lambda_{k} P_{k} \int_{x}^{\infty} y^{-\alpha} y \mathrm{~d} y \\
& =\frac{2 \pi \lambda_{k} P_{k}}{\alpha-2} x^{2-\alpha}
\end{aligned}
$$

where (d) follows from the Campbell's Formula [11]. The integration is done outside the ball $\mathcal{B}(0, x)$ since all the interfering $\mathrm{BSs}$ in the $k$-th tier are farther than $\mathrm{BS}_{k, 0}$, i.e., $\ell_{k, i}>\ell_{k, 0}=x, \forall i \neq 0$.

Plugging (16) into (15) we have

$$
\mathbb{E}_{\Phi_{k}, \mathbf{g}}\left[\log \left(C_{k}\right) \mid \ell_{k, 0}=x\right]=\frac{-2 \pi \tau_{k} \lambda_{k}}{\alpha-2} x^{2} .
$$

Substituting (14) and (17) into (13) and after some manipulations, we have

$$
\mathbb{E}\left[\log \left(C_{k}\right)\right]=\frac{-2 \tau_{k} A_{k}}{\alpha-2} .
$$

By combining (11) and (18) into (10), and plugging back into (9), the average per-user utility is upper bounded by

$$
\begin{aligned}
& U\left(\left\{A_{k}, \eta_{k}\right\}_{\forall k}\right)<\bar{U}\left(\left\{A_{k}, \eta_{k}\right\}_{\forall k}\right) \\
= & \sum_{k=1}^{K} A_{k}\left\{\log \left[\frac{W \eta_{k} \lambda_{k} q_{k} \log \left(1+\tau_{k}\right)}{A_{k} \lambda_{u}}\right]-\frac{2 \tau_{k} A_{k}}{\alpha-2}\right\} .
\end{aligned}
$$

Based on (19), we are able to formulate a maximization of the average user utility upper bound with respect to the user association bias $\left\{B_{k}\right\}_{\forall k}$ and the spectrum partition $\left\{\eta_{k}\right\}_{\forall k}$. However, since the values of bias factors are not unique and we know the association probability and the normalized bias are related as in (3), we optimize $\left\{A_{k}\right\}_{\forall k}$ instead of $\left\{B_{k}\right\}_{\forall k}$.

$$
\begin{array}{ll}
\max _{A_{k}, \eta_{k}, \forall k} & \bar{U}\left(\left\{A_{k}, \eta_{k}\right\}_{\forall k}\right) \\
\text { s.t. } & \sum_{k=1}^{K} A_{k}=1, \\
& \sum_{k=1}^{K} \eta_{k}=1, \\
& A_{k}>0, \eta_{k}>0, \forall k .
\end{array}
$$

\section{B. Optimizing the Spectrum Partition}

Instead of employing a joint optimization directly, we first consider the optimization of spectrum partition only, given that the user association probability $\left\{A_{k}\right\}_{\forall k}$ is fixed.

$$
\begin{array}{ll}
\max _{\eta_{k}, \forall k} & \bar{U}\left(\left\{\eta_{k}\right\}_{\forall k}\right) \\
\text { s.t. } & \sum_{k=1}^{K} \eta_{k}=1, \\
& \eta_{k}>0, \forall k .
\end{array}
$$

By introducing the dual variable $\mu$ with respect to the constraint, we have the Lagrangian

$$
g(\mu)=\bar{U}\left(\left\{\eta_{k}\right\}_{\forall k}\right)-\mu\left(\sum_{k=1}^{K} \eta_{k}-1\right) .
$$

The Karush-Kuhn-Tucker (KKT) condition can be obtained via taking the first order derivative with respect to $\eta_{k}$ as

$$
\frac{A_{k}}{\eta_{k}}-\mu=0 \Rightarrow \eta_{k}^{*}=\frac{A_{k}}{\mu} .
$$


Since $\sum_{k=1}^{K} \eta_{k}^{*}=\frac{\sum_{k=1}^{K} A_{k}}{\mu}=\frac{1}{\mu}=1$, we have $\mu=1$ and consequently the optimal spectrum partition as follows

$$
\eta_{k}^{*}=A_{k} .
$$

The main conclusion here is that the fraction of resources allocated to each tier should be proportional to the number of users associated with that tier, which agrees with intuition.

\section{Optimizing the User Association}

Plugging $\eta_{k}^{*}=A_{k}$ into (19), the joint optimization now turns into an optimization problem over $\left\{A_{k}\right\}_{\forall k}$ only

$$
\begin{array}{ll}
\max _{A_{k}, \forall k} & \bar{U}\left(\left\{A_{k}\right\}_{\forall k}\right) \\
\text { s.t. } & \sum_{k=1}^{K} A_{k}=1, \\
& A_{k}>0, \forall k,
\end{array}
$$

where

$$
\bar{U}\left(\left\{A_{k}\right\}_{\forall k}\right)=\sum_{k=1}^{K} A_{k}\left\{\log \left[\frac{W \lambda_{k} q_{k} \log \left(1+\tau_{k}\right)}{\lambda_{u}}\right]-\frac{2 \tau_{k} A_{k}}{\alpha-2}\right\} .
$$

Problem (25) can be numerically computed using software packages, but it does not have closed form solution because of the complex expression of $q_{k}$ in (11). In wireless systems with a lot more users than base stations, i.e., $\frac{\lambda_{u}}{\lambda_{k}} \gg 1$, the term $\left(1+\frac{A_{k} \lambda_{u}}{3.5 \lambda_{k}}\right)^{-3.5} \approx 0$ and hence $q_{k} \lesssim 1$. We upper bound the utility in (26) assuming $q_{k}=1$ to facilitate our derivation

$$
\begin{aligned}
& \bar{U}\left(\left\{A_{k}\right\}_{\forall k}\right)<\overline{\bar{U}}\left(\left\{A_{k}\right\}_{\forall k}\right) \\
= & \sum_{k=1}^{K} A_{k}\left\{\log \left[\frac{W \lambda_{k} \log \left(1+\tau_{k}\right)}{\lambda_{u}}\right]-\frac{2 \tau_{k} A_{k}}{\alpha-2}\right\} .
\end{aligned}
$$

Modifying problem (25) by maximizing $\overline{\bar{U}}\left(\left\{A_{k}\right\}_{\forall k}\right)$ instead, and employing the dual method we have the Lagrangian

$$
q(\nu)=\overline{\bar{U}}\left(\left\{A_{k}\right\}_{\forall k}\right)-\nu\left(\sum_{k=1}^{K} A_{k}-1\right),
$$

where $\nu$ is the corresponding dual variable. Similarly, the first order condition with respect to $A_{k}$ is

$$
\log \left[\frac{W \lambda_{k} \log \left(1+\tau_{k}\right)}{\lambda_{u}}\right]-\frac{4 \tau_{k} A_{k}}{\alpha-2}-\nu=0,
$$

and the solution to the optimal association is

$$
A_{k}^{*}=\max \left\{\log \left[\frac{W \lambda_{k} \log \left(1+\tau_{k}\right)}{\lambda_{u}}\right]-\nu, 0\right\} \frac{\alpha-2}{4 \tau_{k}},
$$

where $\nu$ should be chosen such that $\sum_{k=1}^{K} A_{k}^{*}=1$ is satisfied. Finally, the corresponding optimal user association bias $B_{k}^{*}$ can be obtained via the transformation (3) based on $A_{k}^{*}$, and the optimal spectrum partition $\eta_{k}^{*}=A_{k}^{*}$.

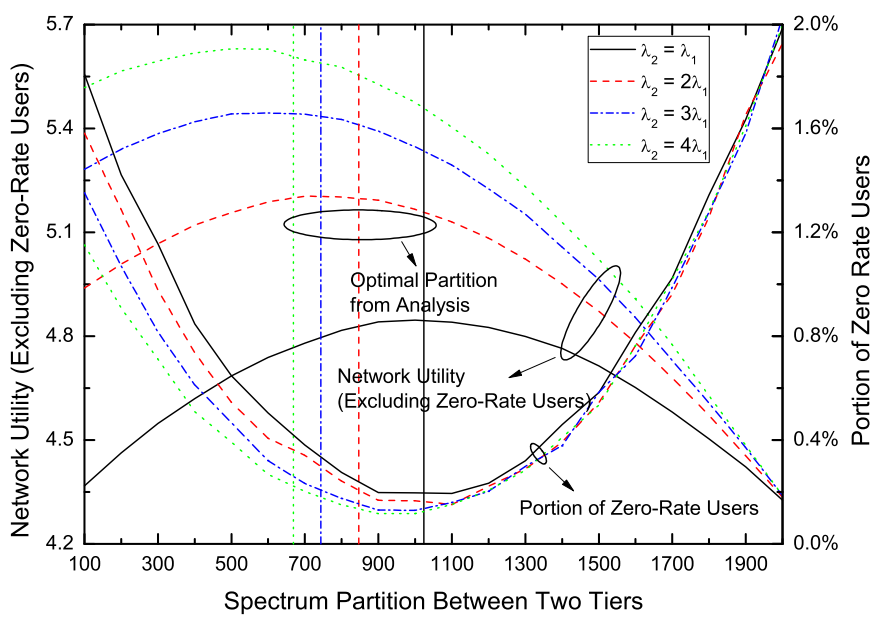

Fig. 2. Network utility of users with non-zero rate, and portion of zero-rate users as a function of spectrum partition between $K=2$ tiers. The $\mathrm{x}$-axis is the subcarriers allocated to the first tier, out of a total of 2048 subcarriers. Other subcarriers are allocated to the second tier. $\left\{P_{1}, P_{2}\right\}=\{46,35\} \mathrm{dBm}$, $\left\{\tau_{1}, \tau_{2}\right\}=\{2,2\}, \lambda_{1}=0.01 \lambda^{(u)}$.

\section{Simulation}

In this section, we present some numerical results on our joint optimization results. In all our simulation, we assume the pathloss exponent $\alpha=4$. The typical user is added at the origin of the 2D plane in addition to the user PPP $\Phi_{u}$. The system bandwidth $W=20 \mathrm{MHz}$ is divided into 2048 subcarriers. Monte Carlo method is applied to generate multiple snapshots of different topologies and channel realizations, and for each snapshot we repeat for 20 time slots. The intensity of the user PPP $\Phi_{u}$ is $\lambda_{u}=\frac{100}{\pi R^{2}}$. The typical user can be scheduled on multiple subcarriers, thus in the simulation the user coverage rate is proportional to the number of subcarriers with SIR larger than the threshold; user get zero rate if SIRs of all of its subcarriers are below the threshold.

First we validate our optimization in a $K=2$ tier network. The transmission powers are $\left\{P_{1}, P_{2}\right\}=\{46,35\} \mathrm{dBm}$. The intensities of the BS PPPs $\Phi_{k}$ are $\lambda_{k}=a_{k} \lambda_{u}$, where $\left\{a_{1}, a_{2}\right\}=\{0.01,0.01 \sim 0.04\}$. The SIR thresholds are set to be $\tau_{k}=2, \forall k$. Since the log-utility is $-\infty$ for users with zero rate, we only compute the average utility of users with non-zero rate, and compute the portion of zero-rate users separately, both of which are plotted in Fig. 2 as a function of the spectrum partition ratio $\eta_{1}$ of the first tier (consequently $\left.\eta_{2}=1-\eta_{1}\right)$. As from (24), we set $A_{k}=\eta_{k}$, and $B_{k}$ can be computed from $A_{k}$ using (3). The optimal $\eta_{k}^{*}$ from analytical solution is also plotted as vertical lines. The optimal spectrum partition should achieve the optimal aggregate utility, thus equivalently it strikes a balance between maximizing the utility of users with non-zero rate and minimizing the zerorate user percentage, as observed from the figure. With a larger tier-2 intensity $\lambda_{2}$, the optimal fraction of resource for tier-1 becomes smaller.

The use of log-utility in the optimization improves the celledge user rate, which is reflected from the 5th-10th percentile 


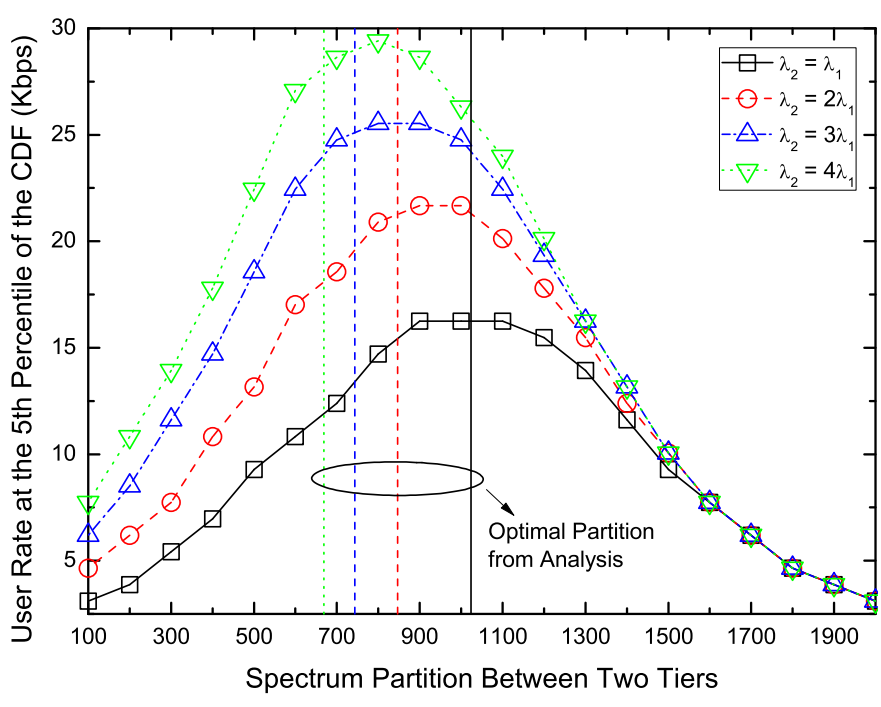

(a)

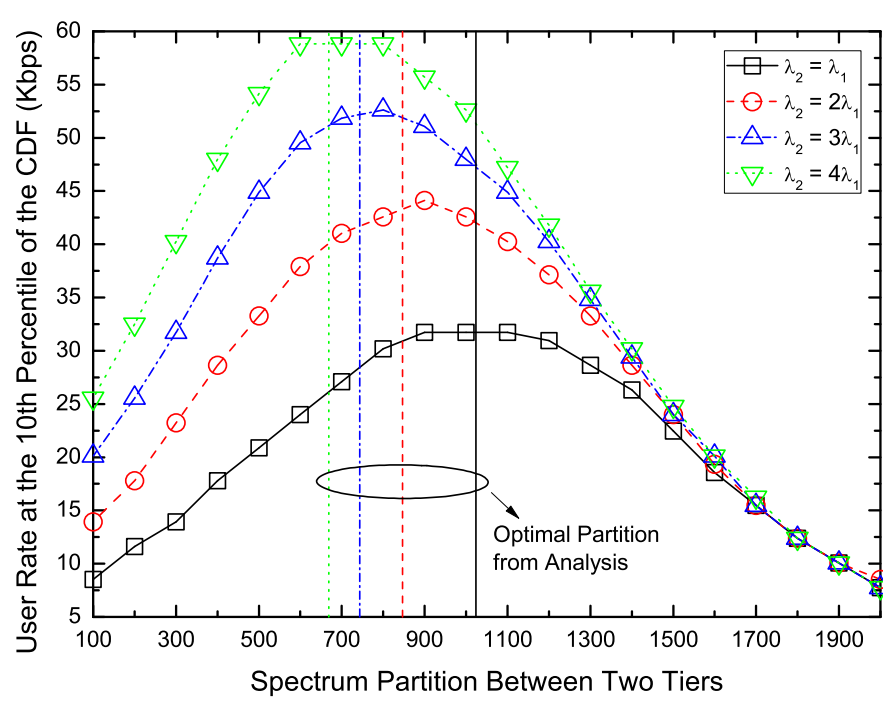

(b)

Fig. 3. The cell-edge user rate as a function of spectrum partition between $K=2$ tiers. The $\mathrm{x}$-axis is the subcarriers allocated to the first tier, out of a total of 2048 subcarriers. Other subcarriers are allocated to the second tier. $\left\{P_{1}, P_{2}\right\}=\{46,35\} \mathrm{dBm},\left\{\tau_{1}, \tau_{2}\right\}=\{2,2\}, \lambda_{1}=0.01 \lambda^{(u)}$. (a) The 5th percentile rate of the CDF. (b) The 10th percentile rate of the CDF.

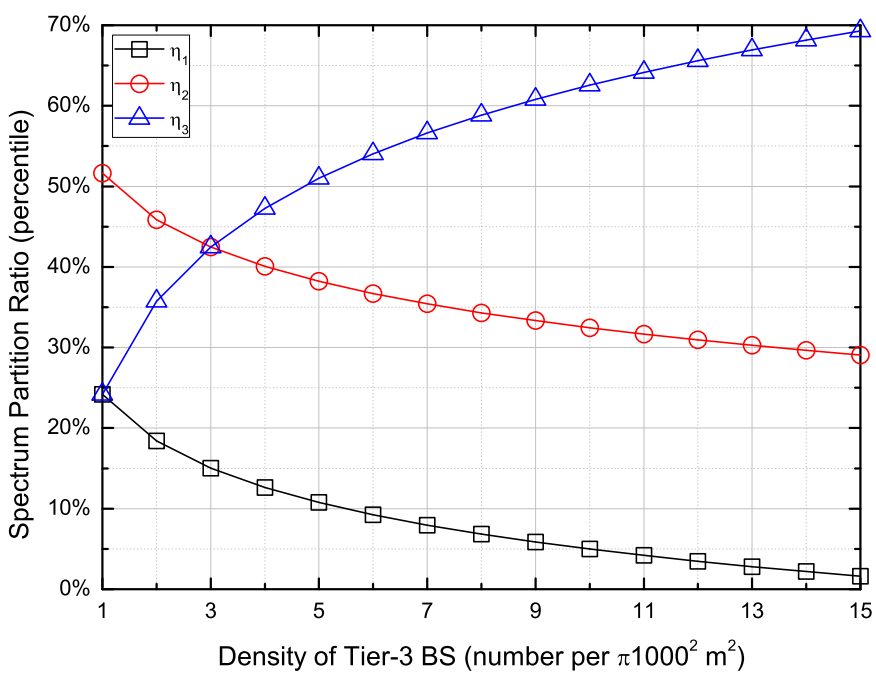

Fig. 4. Spectrum partition ratio as a function of tier-3 BS density. $K=3$, $\left\{P_{1}, P_{2}, P_{3}\right\}=\{46,35,24\} \mathrm{dBm},\left\{\tau_{1}, \tau_{2}, \tau_{3}\right\}=\{2,2,2\},\left\{\lambda_{1}, \lambda_{2}\right\}=$ $\{0.01,0.03\} \lambda^{(u)}$.

point of the user rate CDF. We plot in Fig. 3 the cell-edge rate $(5 \%$ and $10 \%$ respectively) as a function of the spectrum partition ratio $\eta_{1}$. Similar to Fig. 2 , as the tier-2 intensity increases, it is better to allocate less spectrum for tier 1 to improve the cell edge rate. The analytical optimal spectrum partition is found to approximately maximize the user rate at the 10th percentile of the CDF.

In Figs. 4-7, we consider a heterogeneous network with $K=3$ tiers. The transmission powers are $\left\{P_{1}, P_{2}, P_{3}\right\}=$ $\{46,35,24\} \mathrm{dBm}$, BS intensities are $\lambda_{k}=a_{k} \lambda_{u}$ where $\left\{a_{1}, a_{2}, a_{3}\right\}=\{0.01,0.03,0.01 \sim 0.15\}$, and SIR thresholds are $\left\{\tau_{1}, \tau_{2}, \tau_{3}\right\}=\{2,2,1 \sim 11\}$. Figs. $4-5$ show the optimal

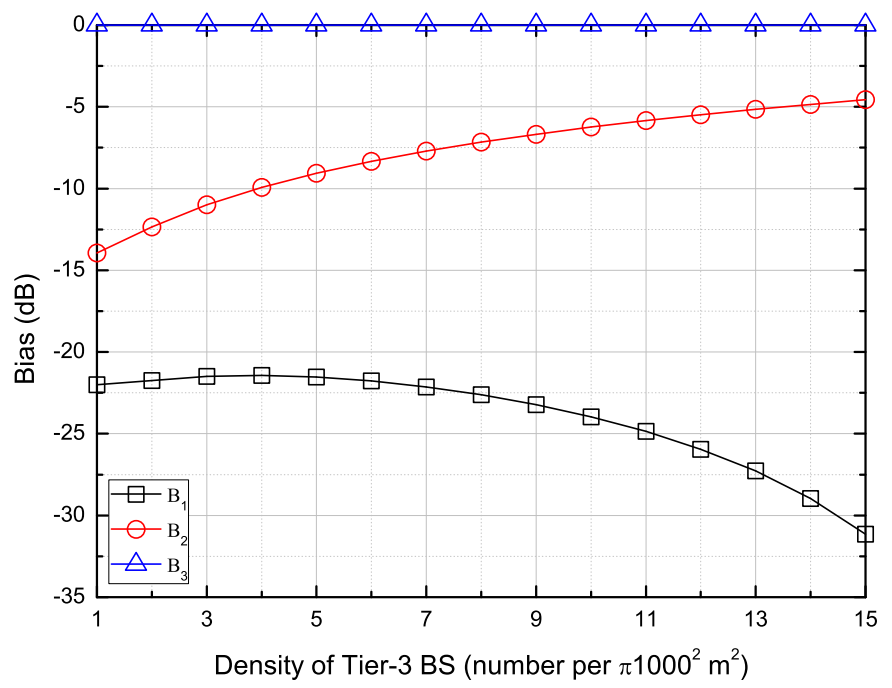

Fig. 5. User association bias as a function of tier-3 BS density. $K=3$, $\left\{P_{1}, P_{2}, P_{3}\right\}=\{46,35,24\} \mathrm{dBm},\left\{\tau_{1}, \tau_{2}, \tau_{3}\right\}=\{2,2,2\},\left\{\lambda_{1}, \lambda_{2}\right\}=$ $\{0.01,0.03\} \lambda^{(u)}$.

spectrum partition and user association bias versus the intensity of the tier-3 BSs. The bias factors are expressed in $\mathrm{dB}$ scale. As more tier-3 BSs are deployed, more users are offloaded to tier-3, hence the fraction of spectrum allocated to tier-3 grows and that of other tiers decreases accordingly. It is interesting to notice that the corresponding bias of tier-1 decreases while the tier-2 bias grows relative to the normalized bias of tier-3 BSs. Figs. 6-7 depict the optimal spectrum partition and user association bias as a function of the SIR threshold. As the minimum SIR of the 3rd tier increases, fewer users choose to associate with tier-3 BSs because of lowered coverage probability, which diminishes its spectrum fraction. After a 


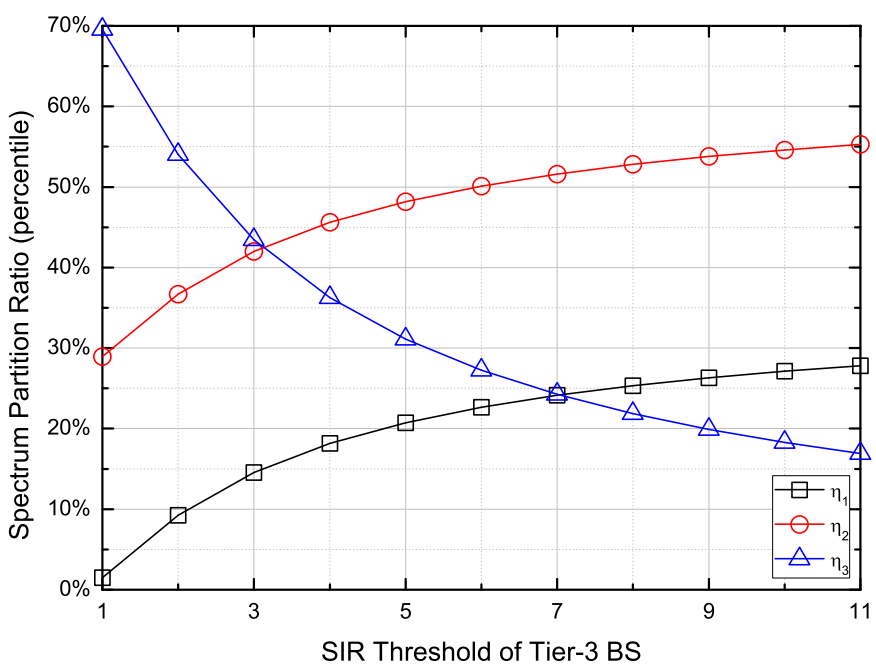

Fig. 6. Spectrum partition ratio as a function of tier-3 BS SIR threshold. $K=$ $3,\left\{P_{1}, P_{2}, P_{3}\right\}=\{46,35,24\} \mathrm{dBm},\left\{\tau_{1}, \tau_{2}\right\}=\{2,2\},\left\{\lambda_{1}, \lambda_{2}, \lambda_{3}\right\}=$ $\{0.01,0.03,0.06\} \lambda^{(u)}$.

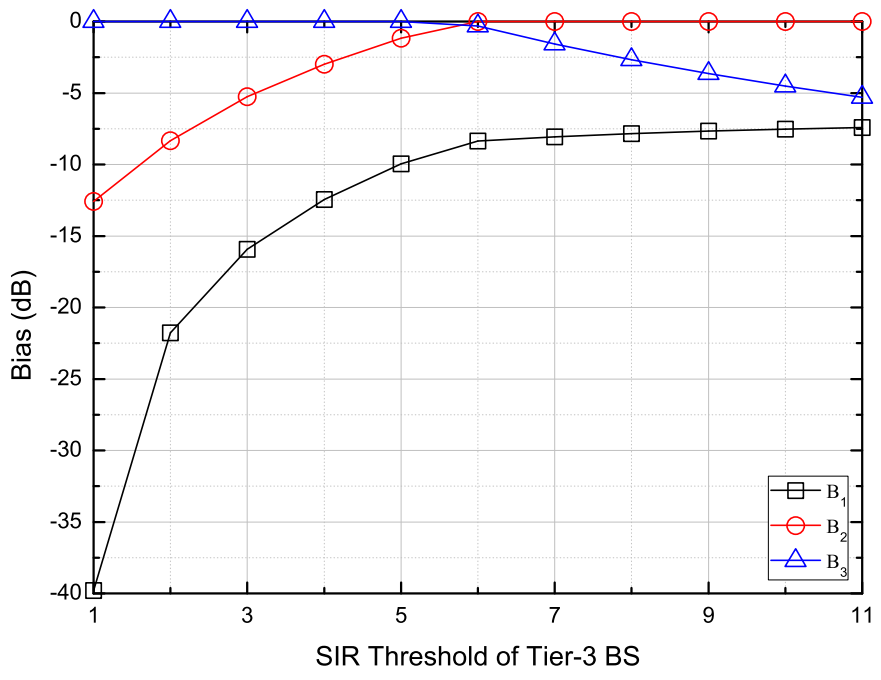

Fig. 7. User association bias as a function of tier-3 BS SIR threshold. $K=$ $3,\left\{P_{1}, P_{2}, P_{3}\right\}=\{46,35,24\} \mathrm{dBm},\left\{\tau_{1}, \tau_{2}\right\}=\{2,2\},\left\{\lambda_{1}, \lambda_{2}, \lambda_{3}\right\}=$ $\{0.01,0.03,0.06\} \lambda^{(u)}$.

certain point $\left(\tau_{3}=6\right.$ in this case), tier-2 BSs take over tier-3 BSs to get the highest bias factor.

\section{CONClusion}

This paper studies the optimal spectrum allocation and user association in heterogeneous multi-tier networks. We first model a stochastic network where all BSs and users are randomly distributed in space, and analytically derive a closedform upper bound of the average proportional fair utility of a typical user using stochastic geometry. A joint optimization framework is then formulated based on this utility, from which the optimal user association bias factor and the optimal fraction of spectrum of each tier are numerically computed. Simulation results verify the accuracy of our analytical result. Our solution also provides useful system insight for heterogeneous networks with different deployment parameters.

\section{REFERENCES}

[1] A. Damnjanovic, J. Montojo, Y. Wei, T. Ji, T. Luo, M. Vajapeyam, T. Yoo, O. Song, and D. Malladi, "A survey on 3GPP heterogeneous networks," IEEE Wireless Commun., vol. 18, no. 3, pp. 10-21, June 2011.

[2] S. Singh, H. Dhillon, and J. Andrews, "Offloading in heterogeneous networks: Modeling, analysis, and design insights," IEEE Trans. Wireless Commun., vol. 12, no. 5, pp. 2484-2497, May 2013.

[3] S. Singh and J. G. Andrews, "Joint resource partitioning and offloading in heterogeneous cellular networks," 2013. [Online]. Available: arXiv: 1303.7039

[4] V. Chandrasekhar and J. Andrews, "Spectrum allocation in tiered cellular networks," IEEE Trans. Commun., vol. 57, no. 10, pp. 3059-3068, Oct. 2009.

[5] W. C. Cheung, T. Q. Quek, and M. Kountouris, "Throughput optimization, spectrum allocation, and access control in two-tier femtocell networks," IEEE J. Sel. Areas Commun., vol. 30, no. 3, pp. 561-574, Apr. 2012

[6] W. Bao and B. Liang, "Structured spectrum allocation and user association in heterogeneous cellular networks," in Proc. IEEE Int. Conf. Comput. Commun. (INFOCOM), June 2014.

[7] Y. Lin and W. Yu, "Optimizing user association and frequency reuse for heterogeneous network under stochastic model," in Proc. IEEE Global Commun. Conf. (GLOBECOM), Dec. 2013.

[8] T. M. Apostol, Mathematical analysis. Addison Wesley Publishing Company, 1974.

[9] H. Jo, Y. Sang, P. Xia, and J. Andrews, "Heterogeneous cellular networks with flexible cell association: A comprehensive downlink SINR analysis," IEEE Trans. Wireless Commun., vol. 11, no. 10, pp. 34843495, Oct. 2012.

[10] S. M. Yu and S.-L. Kim, "Downlink capacity and base station density in cellular networks," in Proc. IEEE WiOpt Workshop on Spatial Stochastic Models for Wireless Networks (SpaSWiN), May 2013.

[11] M. Haenggi, Stochastic Geometry for Wireless Networks. Cambridge University Press, 2012. 